\title{
Observation of vortex coalescence in the anisotropic spin-triplet superconductor $\mathrm{Sr}_{2} \mathrm{RuO}_{4}$
}

\author{
V.O. Dolocan, ${ }^{1}$ C. Veauvy,${ }^{1}$ Y. Liu,${ }^{2}$ F. Servant,${ }^{1}$ P. Lejay, ${ }^{1}$ D. Mailly, ${ }^{3}$ and K. Hasselbach ${ }^{1}$ \\ ${ }^{1}$ CRTBT-CNRS, 25 Avenue des Martyrs, 38042 Grenoble, France \\ ${ }^{2}$ The Pennsylvania State University, University Park, PA 16802,USA \\ ${ }^{3}$ LPN-CNRS, Route de Nozay, 91460 Marcoussis, France
}

(Dated: March 18, 2018)

\begin{abstract}
We present direct imaging of magnetic flux structures in the anisotropic, spin-triplet superconductor $\mathrm{Sr}_{2} \mathrm{RuO}_{4}$ using a scanning $\mu$ SQUID microscope. Individual quantized vortices were seen at low magnetic fields. Coalescing vortices forming flux domains were revealed at intermediate fields. Based on our observations we suggest that a mechanism intrinsic to the material stabilizes the flux domains against the repulsive vortex-vortex interaction. Topological defects like domain walls can provide this, implying proof for unconventional chiral superconductivity.

PACS numbers: 74.20.Rp, 74.25.Qt, 74.70.Pq, 85.25.Dq
\end{abstract}


A Type II superconductor allows flux penetration in the form of quantized vortex lines $\left(\phi_{0}=\mathrm{h} / 2 \mathrm{e}\right)$, when placed in a magnetic field greater than a material and sample-shape dependent lower critical field $\mathrm{H}_{c 1}$. In an isotropic type II superconductor the vortex lines have a shape of round cylinders forming a triangular or square vortex lattice. In the case of anisotropic superconductors vortex shape and vortex lattice structures depend on the symmetry and of the angle of the applied field. $\mathrm{Sr}_{2} \mathrm{RuO}_{4}$ is a tetragonal, layered perovskite superconductor with a superconducting critical temperature $\left(\mathrm{T}_{c}\right)$ of $1.5 \mathrm{~K}[1]$. The anisotropic superconducting properties of $\mathrm{Sr}_{2} \mathrm{RuO}_{4}$ are apparent in the penetration depth anisotropy $\lambda_{c}=3 \mu \mathrm{m}$ and $\lambda_{a b}=0.15 \mu \mathrm{m}$ or in the anisotropy of the critical fields $H_{c 2}^{c}=0.075 \mathrm{~T}$ and $H_{c 2}^{a b}=1.5 \mathrm{~T}[2]$ and are related to its two dimensional Fermi surface.

In the past decade much of the interest on $\mathrm{Sr}_{2} \mathrm{RuO}_{4}$ has derived from the theoretical suggestion [3] and subsequent experimental support [2, 4] that $\mathrm{Sr}_{2} \mathrm{RuO}_{4}$ is a spin-triplet, chiral p-wave superconductor. NMR measurements have observed that the spin susceptibility is unchanged upon entering the superconducting state[5], NQR measurements reveal the absence of a Hebel-Slichter peak in $1 / T_{1} \mathrm{~T}[6]$ and $\mathrm{T}_{c}$ is strongly suppressed by non-magnetic impurities [7]. A spontaneous magnetic field has been detected in the superconducting phase indicating the breaking of the time reversal symmetry (TRS) [8]. A TRS breaking state implies a multiple component order parameter. The microscopic pairing mechanism is still under debate.

Among the possible symmetries of the p-wave state the order parameter $\mathbf{d}(\mathbf{k})=\widehat{z}\left(\mathrm{k}_{x} \pm\right.$ $\left.\mathrm{ik}_{y}\right)$ 9] corresponds closest to the experimental results. The spin of the Cooper pairs lies in the basal plane (equal spin pairing) with the $\mathbf{d}$ vector in the c direction. However this form of $\mathbf{d}$ usually gives a nodeless gap and seems inconsistent with power law dependences observed experimentally in many quantities as for e.g. the specific heat 10, 11]. An interlayer coupling 12] (multi-band model) was proposed to overcome this dilemma along with an orbital dependent superconductivity [13]. The superconductivity originates from an active band $\gamma$ and is induced in the passive bands afterwards through inter-band interaction. The essential order parameter keeps the symmetry $\widehat{z}\left(\mathrm{k}_{x} \pm \mathrm{ik}_{y}\right)$ in the active band with an anisotropic gap.

Degenerate TRS breaking states can appear in the form of domains in the superconduct- 
ing state. Domain walls[14] would separate regions of degenerate order parameters with different surface magnetization. These domains are predicted to act like fences impeding the vortices in their movement. Thus the visualization of vortices and the subsequent observation of intrinsic pinning of vortices [15] are a necessary and important step for resolving the unconventional superconductivity in $\mathrm{Sr}_{2} \mathrm{RuO}_{4}$.

Small angle neutron scattering (SANS) measurements revealed formation of a square vortex lattice 16] in $\mathrm{Sr}_{2} \mathrm{RuO}_{4}$ after field cooling in fields ranging from 50 to 300 gauss applied along c-axis. The square lattice and the detail of the magnetic field distribution around the vortices were found to agree qualitatively with a two-component p-wave Ginzburg-Landau theory [17, 18, 19]. However, SANS is a bulk probe that is sensitive to the long-range correlation in the vortex state rather than to the local structure. No scanning tunneling microscopy images of $\mathrm{Sr}_{2} \mathrm{RuO}_{4}$ succeeded to resolve vortices. Here we present the first microscopic images of the magnetic flux state in $\mathrm{Sr}_{2} \mathrm{RuO}_{4}$, using a custom-built $\mu$ SQUID force microscope $(\mu \mathrm{SFM})[20]$. The $\mu \mathrm{SFM}$ is a sensitive tool for observing individual vortices on a local scale with a spatial resolution of $1 \mu \mathrm{m}$. The $\mu$ SQUID detects the magnetic flux emerging perpendicularly from the sample's surface.

During the imaging, the $\mu$ SQUID moved in a plane above a cleaved ab surface of a single crystal of $\mathrm{Sr}_{2} \mathrm{RuO}_{4}$. The distance between the sample and the SQUID was kept constant at $1 \mu \mathrm{m}$ during scanning by a force detection scheme. The crystal was grown by a floating zone technique using an image furnace[21]. Specific heat measurements of crystals taken from the same single-crystal rod showed volume superconductivity below a temperature of $1.31 \mathrm{~K}$ and a transition width of less than $0.1 \mathrm{~K}$. The sample has a plate like shape with an estimated demagnetizing factor, $\mathrm{N}=0.72$.

Round flux structures are seen after cooling the crystal in a magnetic field of $0.1 \mathrm{G}$ applied along the c-axis, (Fig. 10. Integrating the magnetic field at locations 2 and 3 yields $\sim 1 \phi_{0}$ of flux while the integration at location 1 corresponds to $\sim 2 \phi_{0}$. The measured field profile at locations 2 and 3 can be well adjusted to the model 22] of a single quantized vortex using values for the scanning height between $1-2 \mu \mathrm{m}$ and a penetration depth $\lambda_{a b}=0.15-0.2$ $\mu \mathrm{m}$. The value for the in-plane penetration depth is in agreement with literature values. The quantized amount of flux suggests the presence of single vortices at locations 2 and 3, and a vortex pair at location 1. The flux structure on the bottom right corner, which is not quantized, is most likely due to the presence of a defect at that location. As the 


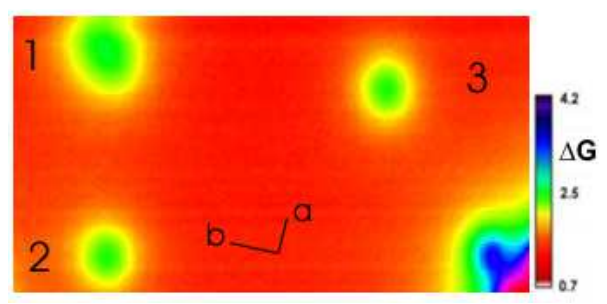

FIG. 1: A $\mu$ SQUID force microscope ( $\mu$ SFM) image of the flux structure above an ab face of $\mathrm{Sr}_{2} \mathrm{RuO}_{4}$, obtained at $\mathrm{T}=0.36 \mathrm{~K}, \mathrm{H}=0.1 \mathrm{G}(\mathrm{H} \| \mathrm{c}$, field cooling at $0.1 \mathrm{G}$ ). The panel corresponds to an imaging size of $31 \mu \mathrm{m} \times 17 \mu \mathrm{m}$. The color legend on the right indicates values of the flux density in gauss. The orientation of the crystallographic a-b axes is shown.

sample temperature decreases below $\mathrm{T}_{c}$ and under applied magnetic field vortices form in a superconductor and may stay pinned at fields lower than $\mathrm{H}_{c 1}$. The observed flux structures were seen to disappear completely above $\mathrm{T}=\mathrm{T}_{c}=(1.35 \pm 0.05) \mathrm{K}$, in agreement with the $\mathrm{T}_{c}$ value determined previously in specific heat measurements.

After field cooling the sample down to $0.35 \mathrm{~K}$ in an applied field of 2 gauss, we observed the presence of flux domains (Fig. 2a). The difference in flux density between the red (normal) and the green (superconducting) regions is 3 gauss. Integration of the flux pattern gives an average field of $1.4 \pm 0.2$ gauss, close to the applied field of 2 gauss. All the flux is condensed in domains, leaving entire superconducting regions empty. These domains are oriented $45^{0}$ from the crystallographic axis. This orientation is in agreement with the flux line lattice orientation observed in SANS and $\mu$ SR. Flux domain structures appear also after zero field cooling (ZFC) the sample and applying 50 gauss at $0.35 \mathrm{~K}$. At equilibrium 5000 vortices should be present at this field and vortices would overlap so much that our SQUID would not resolve them, at best, we expect to detect a weak modulation of the measured flux density. We do observe magnetic field variation as large as 8 gauss between neighboring domains, Fig. 20b.

Vortices in $\mathrm{Sr}_{2} \mathrm{RuO}_{4}$ seem to attract each other and form domains of magnetic flux. A complete collapse of the vortices into one single domain is not observed, probably due to the presence of weak pinning in the material. Domain walls delimiting regions with different order parameters $\left(\mathrm{k}_{x}+\mathrm{ik}_{y}\right.$ and $\left.\mathrm{k}_{x}-\mathrm{i} \mathrm{k}_{y}\right)$ could provide the scenario for weak intrinsic pinning. Domain walls [14] act as pinning regions for the vortices due to the locally diminished condensation energy at the walls. Vortices are fenced in by these walls at low magnetic fields 


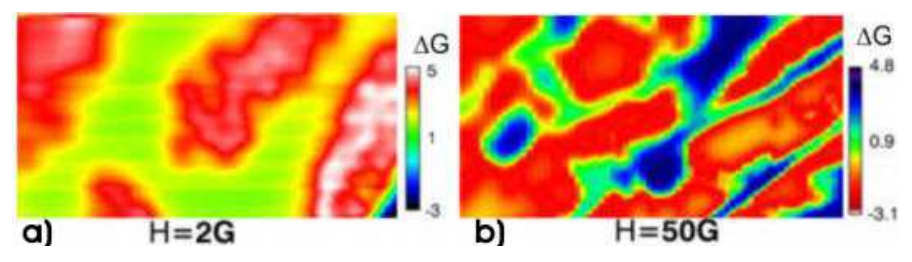

FIG. 2: $\mu$ SQUID imaging of superconducting domain formation in $\mathrm{Sr}_{2} \mathrm{RuO}_{4}$ for magnetic fields applied perpendicular to the ab plane. The data of Fig. 2a) is acquired after field cooling in 2 gauss, the imaging area is $31 \mu \mathrm{m} \times 17 \mu \mathrm{m}$ and flux free domains appear green. Fig. 20 $\mathrm{b}$ ) shows the magnetic state after zero field cooling the sample and applying then 50 gauss, the area is $62 \mu \mathrm{m} \times$ $33 \mu \mathrm{m}$. The measurement temperature is $0.35 \mathrm{~K}$.

and form domains of magnetic flux. In order to examine the stability of the domain configuration the in-plane field was raised while the c-axis field was kept at 2G. Fig. 3] shows for increasing in-plane fields how the condensed vortex structures rearrange freely in order to accommodate the experimental conditions: At 5 gauss in-plane applied field the flux domains become slimmer and above 10 gauss the flux domains are seen to evolve into line-shaped structures. The number of the flux lines was found to increase with the in-plane field in a regular fashion. This regular increase of the flux domain density and their temperature evolution (data not shown) suggest that the flux structures are unrelated to any structural defects in the crystal. Defect pinning [23] of vortices would interfere with regularly spaced vortex pattern (see also ref. 24 and references therein).

The line-shape flux structures resemble vortex chains observed in vortex imaging experiments of $\mathrm{YBa}_{2} \mathrm{Cu}_{3} \mathrm{O}_{7+\delta}[25], \mathrm{Bi}_{2} \mathrm{Sr}_{2} \mathrm{CaCu}_{2} \mathrm{O}_{8+\delta}[26]$ and $\mathrm{NbSe}_{2}$ [27]. Vortex chains appear when the applied field is close to the in-plane direction of the anisotropic superconductor. $\mathrm{Sr}_{2} \mathrm{RuO}_{4}$ has an effective mass anisotropy $(\Gamma) 40$ times higher than $\mathrm{NbSe}_{2}$ and 8 times higher $\mathrm{YBa}_{2} \mathrm{Cu}_{3} \mathrm{O}_{7+\delta}$ but lower than $\mathrm{Bi}_{2} \mathrm{Sr}_{2} \mathrm{CaCu}_{2} \mathrm{O}_{8+\delta}$. Consequently the arrangement of the domains in lines may be driven by the anisotropy, orienting the attractive interaction [28, 29, 30] between vortices along the plane spanned by the anisotropy axis and the in-plane applied field. Nevertheless we observe this coalescence of vortices even when the in-plane field is absent which suggests that another interaction mechanism is present.

How will the flux domain structure evolve as the in-plane field is further tilted toward the anisotropy axis? In Figure 4, the parallel field, $\mathrm{H}_{a b}$, was constant at 10 gauss and the perpendicular component was increased. Each data set was acquired after field cooling. In 


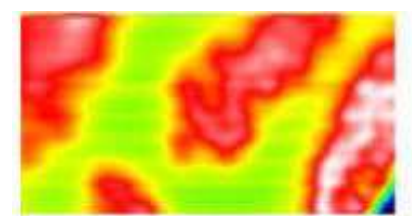

$\mathrm{H}_{\|}=\mathbf{0 G}$

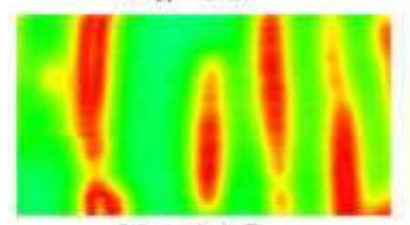

$H_{\|}=10 G$

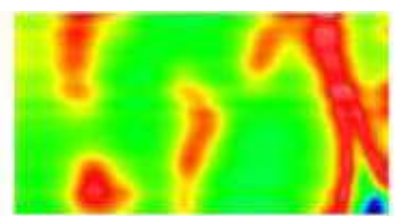

$H_{\|}=5 \mathrm{G}$

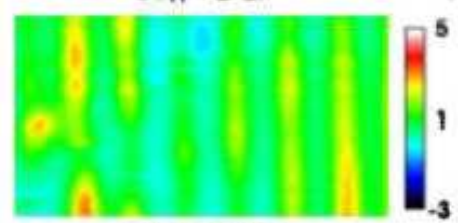

$H_{\|}=50 G$

FIG. 3: $\mu \mathrm{SM}$ images of flux domains in $\mathrm{Sr}_{2} \mathrm{RuO}_{4}$ at $\mathrm{T}=0.36 \mathrm{~K}$ after field cooling at various fields as indicated. The imaging area is $31 \mu \mathrm{m} \times 17 \mu \mathrm{m}$. In all cases, the field amplitude along the c-axis $\left(\mathrm{H}_{\perp}\right)$ was kept constant at $2 \mathrm{G}$ while the in-plane field $\left(\mathrm{H}_{a b}\right)$ was set as indicated. The first panel is identical with the first one in Fig. 2. Field scales in gauss are shown on the right; blue and green regions are flux free regions.

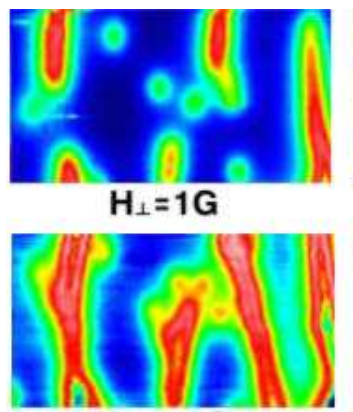

$\mathrm{H}_{\perp}=2.5 \mathrm{G}$

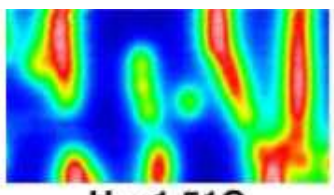

$H_{\perp}=1.51 G$

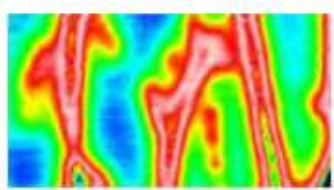

$H_{\perp}=3 G$
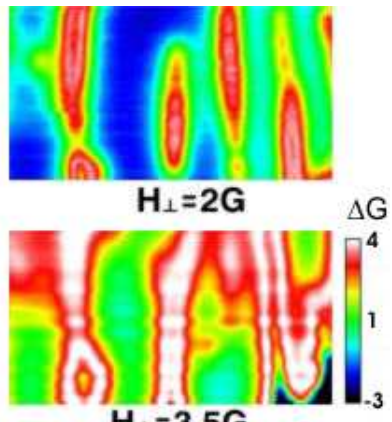

$\mathrm{H}_{\perp}=3.5 \mathrm{G}$

FIG. 4: Magnetic images of the flux structures in $\mathrm{Sr}_{2} \mathrm{RuO}_{4}$ after field cooling keeping $\mathrm{H}_{a b}$ constant at $10 \mathrm{G}$ and $\mathrm{H}_{\perp}$ is varied as indicated. The imaging area is $31 \mu \mathrm{m} \times 17 \mu \mathrm{m}$ and the temperature is $0.36 \mathrm{~K}$. The flux density scale is shown on the right.

the first panel, flux domains and individual vortices are clearly seen. As the perpendicular field was increased more magnetic flux came out of the sample surface. The flux domains attract the individual vortices and at higher fields we see only domains of flux. The domains stretch in the direction of the tilted field. Continuous flux domains are forming for $H_{\perp}$ about 2 gauss. With increasing the field in perpendicular direction the domains are starting to deform and branch, reminiscent of the pattern formation in liquid crystals. For perpendicular fields higher than 2 gauss, broad flux structures split into two very narrow lines staying in 
close proximity. These lines are narrower than individual vortices.

Vortex coalescence overcoming the usual vortex-vortex repulsion[31] is predicted for superconductors with $\kappa$ close to $1 / \sqrt{2}$, the Ginzburg-Landau parameter $\kappa$ is defined as the ratio between the penetration depth and the coherence length. Superconductors with $\kappa<1 / \sqrt{2}$ (type I) don't enter the vortex state but present, in the case of flux penetration, the intermediate state, consisting of meandering domains of normal and superconducting regions. A thin film of a type I superconductor may contain even vortices [32]. An interesting limiting case constitutes Niobium as it is at the border between type I and type II superconductivity. It undergoes a first-oder transition at $H_{c 1}$ accompanied by a magnetization jump $B_{0}$ and consequently presents [33] an intermediate-mixed state if the demagnetizing factor $\mathrm{N} \neq 0$. This state is characterized by the simultaneous presence of flux free regions and regions containing a well-established vortex lattice with a lattice constant corresponding to $B_{0}$. This state exists in a field range between $\mathrm{H}^{*}=H_{c 1}(1-\mathrm{N})$ and $H_{c 1}(1-\mathrm{N})+B_{0} \mathrm{~N}$. Below is the Meissner phase, characterized by the absence of vortices, and above the Shubnikov phase with its vortex lattice. In the intermediate-mixed state an influence of the crystal lattice on the orientation of the flux structures is observed [32]. Generally superconductors with $\kappa>1 / \sqrt{2}$ (type II) have always displayed vortex repulsion and a single quantized vortex state.

In our experimental situation $\mathrm{Sr}_{2} \mathrm{RuO}_{4}$ has a $\kappa$ value $\sim 2$ as the magnetic field is directed along the c-axis, a value significantly higher than $\kappa$ of $\mathrm{Nb}$. No first order transition in the magnetization curves of $\mathrm{Sr}_{2} \mathrm{RuO}_{4}$ is reported. Individual vortices, the signature of type II superconductivity, are present in the sample at low fields after field cooling, but no domains are observed, clearly designating $\mathrm{Sr}_{2} \mathrm{RuO}_{4}$ as a type II superconductor. At intermediate fields the vortices coalesce and leave only a few individual vortices, no vortex lattice is observed. Above $\mathrm{H}_{c 1}$ (35 gauss, estimation based on $\mu$ SQUID measurements) the domains persist, when the vortex lattice formation should have set in. These experimental findings show that the reasons for vortex coalescence in $\mathrm{Sr}_{2} \mathrm{RuO}_{4}$ are different from those in conventional low $\kappa$ superconductors.

The remarkable flux patterns and its systematic variation with the strength and the orientation of the field observed in $\mathrm{Sr}_{2} \mathrm{RuO}_{4}$ can be related solely to intrinsic physical processes in the superconducting state of this material. The coalescence of vortices and splitting of flux domains into very narrow lines are unique features in a type II superconductor and may be due to the presence of domain walls separating regions of different chirality of the order 
parameter. These domain walls would act as corals containing magnetic flux. Furthermore the high mass anisotropy of $\mathrm{Sr}_{2} \mathrm{RuO}_{4}$ will tend to stabilize vortex domains and contributes to regular vortex pattern formation more reminiscent of liquid crystals.

We acknowledge the support of CNRS, and fruitful discussions with V. Mineev, M. Zhitomirsky, M. Sigrist, G. Blatter, V.B. Geshkenbein, and J. Flouquet. Y.L. is supported in part by US ONR.

[1] Y. Maeno et al., Nature(London) 372, 532 (1994).

[2] A. P. Mackenzie and Y. Maeno, Rev. Mod. Phys 75, 657 (2003).

[3] T. M. Rice and M. Sigrist, J. Phys. Condens. Matter 7, 643 (1995).

[4] Y. Maeno, T. M. Rice, and M. Sigrist, Phys. Today 54, 42 (2001).

[5] K. Ishida et al., Nature(London)396, 658 (1994).

[6] K. Ishida et al., Phys. Rev. B 56, 505 (1997).

[7] A. P. Mackenzie et al., Phys. Rev. Lett. 80, 161 (1998).

[8] G. M. Luke et al., Nature(London) 394, 558 (1998).

[9] M. Sigrist and K. Ueda, Rev. Mod. Phys. 63, 239 (1991).

[10] S. Y. Nishizaki, Y. Maeno, and Z. Q. Mao, J. Phys. Soc. Jpn. 69, 572 (2000).

[11] K. Deguchi, Z. Q. Mao, andY. Maeno, J. Phys. Soc. Jpn. 73, 1313 (2004).

[12] M. E. Zhitomirsky and T. M. Rice, Phys. Rev. Lett. 87, 057001 (2001).

[13] D. F. Agterberg, T. M. Rice, and M. Sigrist, Phys. Rev. Lett. 78, 3374 (1997).

[14] M. Sigrist and D. F. Agterberg, Prog. Theor. Phys. 102, 965 (1999).

[15] E. Dumont and A. C. Mota, Phys. Rev. B 65, 144519 (2002).

[16] P. G. Kealey et al., Phys. Rev. Lett. 84, 6094 (2000).

[17] D. F. Agterberg, Phys. Rev. B 58, 14484 (1998).

[18] R. Heeb and D. F. Agterberg, Phys. Rev. B 59, 7076 (1999).

[19] T. Kita, Phys. Rev. Lett. 83, 1846 (1999).

[20] C. Veauvy, D. Mailly, and K. Hasselbach, Rev.Sci.Inst. 73, 3825 (2002).

[21] F. Servant, PhD Thesis, Universite Joseph Fourier, 2002.

[22] J. R. Kirtley, V. G. Kogan, J. R. Clem, and K. A. Moler, Phys. Rev. B 59, 4343 (1999).

[23] D. A. Huse, Phys. Rev. B 46, 8621 (1992). 
[24] C. P. Poole, H. A. Farach, and R. J. Creswick, Superconductivity(Academic, London, 1995), p.294.

[25] P. L. Gammel, D. J. Bishop, J. P. Rice, and D. M. Ginsberg, Phys. Rev. Lett. 68, 3343 (1992).

[26] C. A. Bolle et al., Phys. Rev. Lett. 66, 112 (1991).

[27] H. F. Hess, C. A. Murray, and J. V. Waszczak, Phys. Rev. Lett. 69, 2138 (1992).

[28] A. M. Grishin, A. Yu. Martynovich, S. V. Yampolskii, Zh.Eskp.Teor.Fiz. 97, 1930 (1990) [Sov.Phys.JETP 70, 1089 (1990)].

[29] A. I. Buzdin and A. Yu. Simonov, Zh. Eskp. Teor. Fiz. 98, 2074 (1990) [Sov. Phys. JETP 71, 1165 (1990)].

[30] V. O. Dolocan et al., Physica C 404, 140 (2004).

[31] F. Mohamed, M. Troyer, G. Blatter, and I. Luk'yanchuk, Phys. Rev. B 65, 224504 (2002).

[32] R. P. Hubner, Magnetic Flux structures in Superconductors(Springer, Berlin, 1979), p.75.

[33] H.R. Kerchner, D.K. Christen, and S.T. Sekula, Phys. Rev. B. 21, 86 (1980). 